\title{
EDITORIAL Biological and behavioural antidepressant treatment responses with the selective serotonin reuptake inhibitor fluoxetine can be determined by the environment
}

Molecular Psychiatry (2017) 22, 484; doi:10.1038/mp.2017.68

Given the effects of fluoxetine (FLX) on the brain and depression described by Alboni et al., ${ }^{1}$ the role of the environment should be considered by those using either this drug or possibly other selective serotonin reuptake inhibitors (SSRI's). Despite SSRIs being the most prescribed treatment for depression, the variability of their effectiveness is high, as $\sim 60-70 \%$ of patients do not experience remission and $30-40 \%$ do not show a significant response. The paper by Alboni et al. elucidates what this might be due to.

The role of serotonin in depression has been extensively studied by various approaches, including acute tryptophan depression. ${ }^{2}$ The development of SSRIs has been an important milestone in the treatment of depression, as they have significantly fewer side effects than previous drug classes. In particular, SSRIs do not cause cardiac conduction abnormalities in overdose and have a low propensity to cause seizures. FLX was the first SSRI introduced in the USA (1988). ${ }^{3,4}$ We showed that the increase in prescriptions in FLX in the USA from 1988 to 2002 was correlated with decreased rates of suicides and suggested that as untreated depression is major cause of suicide, FLX treatment could have had a contributory role in the reduction of suicide rates in the period $1988-2002 .^{5}$

Despite its wide usage, the mechanism of action of SSRIs is poorly understood. An emerging hypothesis is that SSRIs increase serotonin levels, enhance neural plasticity, allowing for the environment to more readily influence the individual. Given this, a rich, positive environment should correlate to a reduction of symptoms and conversely, in a stressful environment, the symptoms would worsen.

This hypothesis was rigorously tested on C56BL/6 mice that were induced to become phenotypically depressed because they were exposed to chronic stress for 2 weeks. As expected, this significantly induced anhedonic behaviour and increased corticosterone levels. Mice were then treated for 3 weeks in either enriched or stressed conditions, with comparisons between vehicle (VEH)- vs FLX-treated mice. FLX was found to have opposite outcomes in those two different environments. Such effects were evidenced not only in behaviour but also in the levels of hypothalamic-pituitary-adrenal (HPA) axis activity. Corticosterone levels were reduced by FLX in the enriched but not in the stressful condition. In the enriched condition, glucocorticoid receptor (GR) and mineralocorticoid receptor messenger RNA expression were not changed; FLX reduced GR, but not mineralocorticoid receptor, messenger RNA levels in the stressful condition, potentially resulting in blunting of HPA feedback, which would explain the lack of reduction in corticosterone levels in mice exposed to stress following FLX.

FLX had no effect on neurogenesis in the enriched condition, but caused detrimental consequences on neuronal progenitor proliferation in the stressful condition. The number of proliferating cells was reduced across the entire hippocampus in FLX when compared to VEH mice in the stressful condition. BDNF expression was found to be increased in FLX in the enriched condition and high levels have been reported to increase p11 expression, a protein involved in depression and antidepressant response. Increased levels of p11 messenger RNA were also found in the hippocampus of FLX compared with VEH mice in the enriched but not in the stressful condition. ERK, CREB and BDNF protein levels in the medial prefrontal cortex were also found to be in line with the hypothesis of the study.

The causation of depression is the outcome of gene-environment interactions. ${ }^{6}$ What is paradigm shifting in the paper by Alboni et al. ${ }^{1}$ is the meticulous demonstration that the responses to antidepressant treatment are the result of drug-environmental interactions with opposite outcomes, depending on the type of environment that the treated animal is exposed to. It is now imperative to translate this novel concept by testing the hypothesis that the variability in the outcomes of SSRI treatment of depression in humans is due, at least in part, to environmental factors. There is now compelling evidence that the environment is critical to SSRI response. As it is the case for depression causation, it can be suggested, based on the remarkable work of Alboni et al. $^{1}$ and on a body of work on pharmacogenomics, ${ }^{7}$ that antidepressant treatment response is likewise the outcome of gene-environment interactions.

Further studies should not only to translate these findings to humans, but they will also need to ascertain whether the effects observed by Alboni et al. are restricted to fluoxetine or if they can also be carried by other SSRIs or possibly by other classes of antidepressant drugs. It would also be valuable to determine if these remarkably varying effects may also occur after other types of depression treatment, such as transcranial magnetic stimulation (TMS).

\section{CONFLICT OF INTEREST}

The authors declare no conflict of interest.

AW Licinio $^{1,2}$, ML Wong $^{1}$ and J Licinio ${ }^{1}$ ${ }^{1}$ Mind \& Brain Theme, South Australian Health and Medical Research Institute and Department of Psychiatry, School of Medicine, Flinders University, Adelaide, SA, Australia and ${ }^{2}$ University Vita-Salute San Raffaele, Milano, Italy E-mail: julio.licinio@sahmri.com

\section{REFERENCES}

1 Alboni S, van Dijk RM, Poggini S, Milior G, Perrotta M, Drenth $\mathrm{T}$ et al. Mol Psychiatry 2017; 22: 552-561 (this issue).

2 Miller HL, Delgado PL, Salomon RM, Licinio J, Barr LC, Charney DS. J Clin Psychiatry 1992; 53 (Suppl): 28-35.

3 Stokes PE. Clin Ther 1993; 15: 216-243.

4 Wong ML, Dong C, Flores DL. Am J Psychiatry 2014; 171: 1297-1309.

5 Milane MS, Suchard MA, Wong ML, Licinio J. PLoS Med 2006; 3: e190.

6 Wong ML, Dong C, Andreev V, Arcos-Burgos M, Licinio J. Mol Psychiatry 2012; 17: 624-633.

7 Licinio J, Wong ML. Pharmacogenomics: the Search for Individualized Therapies. John Wiley \& Sons: Weinheim, Germany, 2002. 